\title{
Cloud Computing: Benefits, Risks and Recommendations for Information Security
}

\author{
Daniele Catteddu \\ ENISA, Greece \\ Daniele.Catteddu@enisa. europa.eu
}

The presentation "Cloud Computing: Benefits, risks and recommendations for information security" will cover some the most relevant information security implications of cloud computing from the technical, policy and legal perspective.

Information security benefit and top risks will be outlined and most importantly, concrete recommendations for how to address the risks and maximise the benefits for users will be given. 